\title{
The impact of inflation risk on forward trading and production
}

\author{
Udo Broll \\ Technische Universität Dresden \\ Kit Pong Wong \\ University of Hong Kong
}

September 2015

\begin{abstract}
This paper examines the behavior of a competitive firm that faces joint price and inflation risk. Given that the price risk is negatively correlated with the inflation risk in the sense of expectation dependence, we show that the firm optimally opts for an over-hedge (under-hedge) if the firm's coefficient of relative risk aversion is everywhere no greater (no smaller) than unity. We show further that banning the firm from forward trading may induce the firm to produce more or less, depending on whether the price risk premium is positive or negative, respectively. While the price risk premium is unambiguously negative in the absence of the inflation risk, it is not the case when the inflation risk prevails. In contrast to the conventional wisdom, forward hedging needs not always promote production should firms take inflation seriously.
\end{abstract}

JEL classification: D21; D24; D81

Keywords: Forward hedging; Expectation dependence; Inflation risk; Production 


\section{The impact of inflation risk on forward trading and production}

This paper examines the behavior of a competitive firm that faces joint price and inflation risk. Given that the price risk is negatively correlated with the inflation risk in the sense of expectation dependence, we show that the firm optimally opts for an over-hedge (underhedge) if the firm's coefficient of relative risk aversion is everywhere no greater (no smaller) than unity. We show further that banning the firm from forward trading may induce the firm to produce more or less, depending on whether the price risk premium is positive or negative, respectively. While the price risk premium is unambiguously negative in the absence of the inflation risk, it is not the case when the inflation risk prevails. In contrast to the conventional wisdom, forward hedging needs not always promote production should firms take inflation seriously.

\section{Introduction}

The importance of risk management has inspired an extensive literature on investment, production, and consumption under uncertainty. Most of the extant studies have made the implicit assumption that decision makers care only about their random nominal wealth. Notable exceptions are Adam-Müller (2002a, 2002b) and Battermann and Broll (2001), which examine the production and hedging decisions of the competitive firm $\grave{a}$ la Sandmo (1971) when real wealth matters. In the presence of inflation risk, the celebrated separation theorem remains intact while the full-hedging theorem no long holds. Adam-Müller (2002a) shows further that banning the firm from forward trading may induce the firm to produce more, not less, a rather striking result that does not arise if there is no inflation risk.

The purpose of this paper is to complement the results of Adam-Müller (2002). To characterize the firm's optimal forward position, we show that the concept of expectation dependence (Wright, 1987) is useful. ${ }^{1}$ While Adam-Müller (2002) specifies the inflation risk as a monotonically decreasing function of the price risk plus noise, expectation dependence

\footnotetext{
${ }^{1}$ For more applications, see Broll et al. (2015), Li (2011), and Wong (2012, 2013).
} 
provides an alternative bivariate dependence structure. Given that the price risk is negatively correlated with the inflation risk in the sense of expectation dependence, we show that the firm optimally opts for an over-hedge (under-hedge) should the firm's coefficient of relative risk aversion be everywhere no greater (no smaller) than unity, which is consistent with the results of Adam-Müller (2002). We show further that the firm optimally produces more or less in the absence than in the presence of forward hedging, depending on whether the price risk premium is positive or negative, respectively. In the absence of the inflation risk, the price risk premium is always negative, thereby rendering the adverse effect on output when forward trading is not allowed (see, for example, Holthausen 1979). When the inflation risk prevails, the price risk premium can be positive so that forward trading may not promote production, a stark contrast to the conventional wisdom.

The rest of this paper is organized as follows. Section 2 delineates the model of the competitive firm under joint price and inflation risk. Section 3 introduces the concept of expectation dependence. Section 4 characterizes the firm's optimal forward position. Section 5 examines the firm's optimal production decision. The final section concludes.

\section{The model}

Consider a competitive firm that operates for one period with two dates, 0 and 1 . To begin, the firm acquires inputs at known nominal prices to produce a single commodity. The nominal value of inputs at date 1 gives rise to a deterministic cost function, $C(Q)$, where $Q \geq 0$ is the output level chosen by the firm at date $0, C(0)=C^{\prime}(0)=0$, and $C^{\prime}(Q)>0$ and $C^{\prime \prime}(Q)>0$ for all $Q>0 .^{2}$

At date 1, the firm sells its entire output, $Q$, at the uncertain nominal output price,

$\tilde{P}{ }^{3}$ We model inflation risk by a stochastic purchasing power index, $\tilde{Z}$, with unit mean so

\footnotetext{
${ }^{2}$ The strict convexity of $C(Q)$ is driven by the firm's production technology that exhibits decreasing returns to scale.

${ }^{3}$ Throughout the paper, random variables have a tilde $(\sim)$ while their realizations do not.
} 
that $\tilde{Z}-1$ gauges surprises due to purchasing power changes. Let $F(P)$ be the marginal cumulative distribution function (CDF) of $\tilde{P}$ over support $[\underline{P}, \bar{P}]$ with $0<\underline{P}<\bar{P}$. Likewise, let $G(Z)$ be the marginal CDF of $\tilde{Z}$ over support $[\underline{Z}, \bar{Z}]$ with $0<\underline{Z}<\bar{Z}$. To allow the price risk, $\tilde{P}$, to be correlated with the inflation risk, $\tilde{Z}$, we denote $H(P, Z)$ as their joint CDF over support $[\underline{P}, \bar{P}] \times[\underline{Z}, \bar{Z}]$.

While the inflation risk, $\tilde{Z}$, is neither hedgeable nor insurable, the firm can hedge against the price risk, $\tilde{P}$, by selling (purchasing if negative) $X$ units of its output forward at the known forward price, $P^{f}$, at date 0 . The firm's real income at date 1 is, therefore, given by

$$
\tilde{\Pi}=\tilde{Z}\left[W+\tilde{P} Q+\left(P^{f}-\tilde{P}\right) X-C(Q)\right]
$$

where $W>0$ is a fixed component of nominal income. We say that the firm's forward position, $X$, is an under-hedge, a full-hedge, or an over-hedge, depending on whether $X$ is smaller than, equal to, or greater than the output level, $Q$, respectively.

The firm is risk averse and possesses a von Neumann-Morgenstern utility function, $U(\Pi)$, defined over its real income at date 1 , $\Pi$, with $U^{\prime}(\Pi)>0$ and $U^{\prime \prime}(\Pi)<0$ for all $\Pi>0$. The firm's ex-ante decision problem at date 0 is to choose its output level, $Q$, and its forward position, $X$, so as to maximize the expected utility of its real income at date 1 :

$$
\max _{Q \geq 0, X} \mathrm{E}[U(\tilde{\Pi})]
$$

where $\mathrm{E}(\cdot)$ is the expectation operator with respect to $H(P, Z)$, and $\tilde{\Pi}$ is given by Eq. (1).

The first-order conditions for program (2) are given by

$$
\mathrm{E}\left\{U^{\prime}\left(\tilde{\Pi}^{*}\right) \tilde{Z}\left[\tilde{P}-C^{\prime}\left(Q^{*}\right)\right]\right\}=0
$$

and

$$
\mathrm{E}\left[U^{\prime}\left(\tilde{\Pi}^{*}\right) \tilde{Z}\left(P^{f}-\tilde{P}\right)\right]=0
$$

where an asterisk $\left(^{*}\right)$ signifies an optimal level. The second-order conditions for program (2) are satisfied given that $U^{\prime \prime}(\Pi)<0$ and $C^{\prime \prime}(Q)>0$. We restrict our attention to the case that the forward price is unbiased, i.e., $P^{f}=\mathrm{E}(\tilde{P})$. 


\section{Expectation dependence}

We define the CDF of $\tilde{P}$ conditional on the event that $\tilde{Z} \leq Z$ as

$$
F(P \mid \tilde{Z} \leq Z)=\frac{H(P, Z)}{G(Z)}
$$

over support $[\underline{P}, \bar{P}]$ for all $Z \in[\underline{Z}, \bar{Z}]$. Let $\mathrm{E}(\tilde{P} \mid \tilde{Z} \leq Z)$ be the expected value of $\tilde{P}$ with respect to the conditional $\mathrm{CDF}, F(P \mid \tilde{Z} \leq Z)$. The following bivariate dependence structure, known as expectation dependence, is due to Wright (1987).

Definition 1 The price risk, $\tilde{P}$, is said to be negatively (positively) expectation dependent on the inflation risk, $\tilde{Z}$, if

$$
\mathrm{ED}(\tilde{P} \mid Z)=\mathrm{E}(\tilde{P})-\mathrm{E}(\tilde{P} \mid \tilde{Z} \leq Z) \leq(\geq) 0
$$

for all $Z \in[\underline{Z}, \bar{Z}]$, where the inequality is strict for some non-degenerate intervals.

Eq. (6) implies that the expected value of the price risk, $\tilde{P}$, is revised downward (upward) whenever the inflation risk, $\tilde{Z}$, is discovered to be small, in the precise sense that the truncation, $\tilde{Z} \leq Z$, is presented. To see further how Definition 1 defines dependence, we write Eq. (6) as

$$
\begin{aligned}
\mathrm{ED}(\tilde{P} \mid Z) & =\int_{\underline{P}}^{\bar{P}} P \mathrm{~d} F(P)-\int_{\underline{P}}^{\bar{P}} P \mathrm{~d} F(P \mid \tilde{Z} \leq Z) \\
& =\int_{\underline{P}}^{\bar{P}}[F(P \mid \tilde{Z} \leq Z)-F(P)] \mathrm{d} P,
\end{aligned}
$$

where the second equality follows from integration by parts. It is evident from Eq. (8) that $\operatorname{ED}(\tilde{P} \mid Z) \leq(\geq) 0$ if $F(P \mid \tilde{Z} \leq Z) \leq(\geq) F(P)$ for all $P \in[\underline{P}, \bar{P}]$, i.e., if $F(P \mid \tilde{Z} \leq Z)$ dominates (is dominated by) $F(P)$ in the sense of first-order stochastic dominance. Hence, negative (positive) expectation dependence is implied by the fact that small inflation risk decreases (increases) the price risk in the sense of first-order stochastic dominance, which can be verified empirically using tests of stochastic dominance. Wright (1987) shows that 
negative (positive) expectation dependence is a sufficient, but not necessary, condition for negative (positive) correlation.

Adam-Müller (2002) specifies the inflation risk as $\tilde{Z}=b(\tilde{P})+\tilde{\epsilon}$, where $b(P)$ is a deterministic function with $b^{\prime}(P)<0$, and $\tilde{\epsilon}$ is a zero-mean random variable such that the price risk, $\tilde{P}$, is conditionally independent of $\tilde{\epsilon}$, i.e., $\mathrm{E}(\tilde{P} \mid \epsilon)=\mathrm{E}(\tilde{P})$ for all $\epsilon$. An alternative specification along the lines of Adam-Müller (2002) is to model the price risk as $\tilde{P}=B(\tilde{Z})+\tilde{\eta}$, where $B(Z)$ is a deterministic function with $B^{\prime}(Z)<0$, and $\tilde{\eta}$ is a zero-mean random variable such that the inflation risk, $\tilde{Z}$, is conditionally independent of $\tilde{\eta}$, i.e., $\mathrm{E}(\tilde{Z} \mid \eta)=\mathrm{E}(\tilde{Z})$ for all $\eta$. This alternative specification captures a more intuitive idea that the output price, $\tilde{P}$, is directly, albeit imperfectly, influenced by the purchasing power index, $Z$, through the deterministic function, $B(Z)$. Indeed, it is easily verified that these two different specifications give rise to observationally equivalent results within the context of Adam-Müller (2002).

Suppose that $\tilde{P}=B(\tilde{Z})+\tilde{\eta}$. We can write Eq. (6) as

$$
\operatorname{ED}(\tilde{P} \mid Z)=\int_{\underline{Z}}^{\bar{Z}} B(z) \mathrm{d} G(z)-\int_{\underline{Z}}^{Z} \frac{B(z)}{G(Z)} \mathrm{d} G(z),
$$

for all $Z \in[\underline{Z}, \bar{Z}]$, since $\tilde{Z}$ is conditionally independent of $\tilde{\eta}$. Differentiating Eq. (8) with respect to $Z$ yields

$$
\frac{\partial \mathrm{ED}(\tilde{P} \mid Z)}{\partial Z}=\frac{G^{\prime}(Z)}{G(Z)^{2}} \int_{\underline{Z}}^{Z}[B(z)-B(Z)] \mathrm{d} G(z) .
$$

Define the following function:

$$
\Phi(Z)=\int_{\underline{Z}}^{Z}[B(z)-B(Z)] \mathrm{d} G(z)
$$

Differentiating Eq. (10) with respect to $Z$, we have $\Phi^{\prime}(Z)=-B^{\prime}(Z) G(Z)$. Consider first the case that $B^{\prime}(Z)<0$ so that $\Phi^{\prime}(Z)>0$. Since $\Phi(\underline{Z})=0$, it follows that $\Phi(Z)>0$ and the left-hand side of Eq. (9) is positive for all $Z \in(\underline{Z}, \bar{Z}]$. Since $\operatorname{ED}(\tilde{P} \mid \bar{Z})=0$, it follows that $\operatorname{ED}(\tilde{P} \mid Z)<0$ for all $Z \in[\underline{Z}, \bar{Z})$. Hence, the alternative specification along the lines of Adam-Müller (2002) implies that $\tilde{P}$ is negatively expectation dependent on $\tilde{Z}$. 
We now consider the case that $B(Z)$ is $\mathrm{U}$-shaped such that $B(\bar{Z}) \leq \mathrm{E}[B(\tilde{Z})]$. It follows from $\Phi^{\prime}(Z)=-B^{\prime}(Z) G(Z)$ that $\Phi(Z)$ has an inverted U-shape. Since $B(\bar{Z}) \leq \mathrm{E}[B(\tilde{Z})]$, we have $\Phi(\bar{Z}) \geq 0$ from Eq. (10). Given that $\Phi(\underline{Z})$, it must be true that $\Phi(Z)>0$ and the left-hand side of Eq. (9) is positive for all $Z \in(\underline{Z}, \bar{Z})$. Since $\operatorname{ED}(\tilde{P} \mid \bar{Z})=0$, it follows that $\operatorname{ED}(\tilde{P} \mid Z)<0$ for all $Z \in[\underline{Z}, \bar{Z})$. Although $\tilde{P}$ and $\tilde{Z}$ do not follow the alternative specification along the lines of Adam-Müller (2002), $\tilde{P}$ is negatively expectation dependent on $\tilde{Z}$. In light of this, expectation dependence provides a more general dependence structure than the one used by Adam-Müller (2002). We as such focus hereafter on the case that $\tilde{P}$ and $\tilde{Z}$ are negatively expectation dependent. ${ }^{4}$

\section{Optimal hedging decision}

In this section, we characterize the firm's optimal forward position, $X^{*}$. To this end, we use the covariance operator, $\operatorname{Cov}(\cdot, \cdot)$, with respect to $H(P, Z)$ to write Eq. (4) as ${ }^{5}$

$$
\operatorname{Cov}\left[U^{\prime}\left(\tilde{\Pi}^{*}\right) \tilde{Z}, \tilde{P}\right]=0
$$

since $P^{f}=\mathrm{E}(\tilde{P})$. Differentiating $\mathrm{E}[U(\tilde{\Pi})]$ with respect to $X$ and evaluating the resulting derivative at $Q=Q^{*}$ and $X=Q^{*}$ yields

$$
\left.\frac{\partial \mathrm{E}[U(\tilde{\Pi})]}{\partial X}\right|_{Q=Q^{*}, X=Q^{*}}=-\operatorname{Cov}\left\{U^{\prime}[\Pi(\tilde{Z})] \tilde{Z}, \tilde{P}\right\},
$$

where $\Pi(\tilde{Z})=\tilde{Z}\left[W+\mathrm{E}(\tilde{P}) Q^{*}-C\left(Q^{*}\right)\right]$. If the right-hand side of Eq. (12) is positive (negative), it follows immediately from Eq. (11) and the second-order conditions for program (2) that $X^{*}>(<) Q^{*}$.

Cuadras (2002) proves that $\operatorname{Cov}[\alpha(\tilde{P}), \beta(\tilde{Z})]$ can be written in terms of the CDFs, $F(P)$, $G(Z)$, and $H(P, Z)$, as follows:

$$
\operatorname{Cov}[\alpha(\tilde{P}), \beta(\tilde{Z})]=\int_{\underline{P}}^{\bar{P}} \int_{\underline{Z}}^{\bar{Z}}[H(P, Z)-F(P) G(Z)] \mathrm{d} \alpha(P) \mathrm{d} \beta(Z),
$$

\footnotetext{
${ }^{4}$ The less likely case wherein $\tilde{P}$ and $\tilde{Z}$ are positively expectation dependent can be analogously analyzed.

${ }^{5}$ For any two random variables, $\tilde{X}$ and $\tilde{Y}$, it is true that $\operatorname{Cov}(\tilde{X}, \tilde{Y})=\mathrm{E}(\tilde{X} \tilde{Y})-\mathrm{E}(\tilde{X}) \mathrm{E}(\tilde{Y})$.
} 
where $\alpha(\cdot)$ and $\beta(\cdot)$ are functions of bounded variation. Using Eq. (13) with $\alpha(\tilde{P})=\tilde{P}$ and $\beta(\tilde{Z})=U^{\prime}[\Pi(\tilde{Z})] \tilde{Z}$, we can write the right-hand side of Eq. (12) as

$$
\begin{gathered}
-\int_{\underline{P}}^{\bar{P}} \int_{\underline{Z}}^{\bar{Z}}[H(P, Z)-F(P) G(Z)]\left\{U^{\prime}[\Pi(Z)]+U^{\prime \prime}[\Pi(Z)] \Pi(Z)\right\} \mathrm{d} P \mathrm{~d} Z \\
=-\int_{\underline{Z}}^{\bar{Z}} \operatorname{ED}(\tilde{P} \mid Z)\{1-R[\Pi(Z)]\} U^{\prime}[\Pi(Z)] G(Z) \mathrm{d} Z
\end{gathered}
$$

where $\operatorname{ED}(\tilde{P} \mid Z)$ is defined in Eq. (6), and $R(\Pi)=-\Pi U^{\prime \prime}(\Pi) / U^{\prime}(\Pi)$ for all $\Pi>0$ is the Arrow-Pratt measure of relative risk aversion. Since $\tilde{P}$ is negatively expectation dependent on $\tilde{Z}$, we have $\operatorname{ED}(\tilde{P} \mid Z) \leq 0$ for all $Z \in[\underline{Z}, \bar{Z}]$. The right-hand side of Eq. (14) is positive (negative) if $R(\Pi) \leq(\geq) 1$ for all $\Pi>0$, thereby invoking the following proposition. ${ }^{6}$

Proposition 1 Given that the price risk, $\tilde{P}$, is negatively expectation dependent on the inflation risk, $\tilde{Z}$, the competitive firm that can sell its output forward at the unbiased forward price, $P^{f}=\mathrm{E}(\tilde{P})$, optimally opts for an over-hedge (under-hedge), i.e., $X^{*}>(<) Q^{*}$, if the Arrow-Pratt measure of relative risk aversion, $R(\Pi)=-\Pi U^{\prime \prime}(\Pi) / U^{\prime}(\Pi)$, is everywhere no greater (no smaller) than unity.

The intuition for Proposition 1 is as follows. Eq. (11) implies that the optimal forward position, $X^{*}$, is the one that makes the multiple of the firm's marginal utility, $U^{\prime}\left(\tilde{\Pi}^{*}\right)$, and the inflation risk, $\tilde{Z}$, invariant to the price risk, $\tilde{P}$. Since $\tilde{P}$ and $\tilde{Z}$ are negatively correlated in the sense of expectation dependence, they are natural hedges against each other. Starting with a full-hedge, the firm has a cross-hedging incentive that reduces the firm's forward position. To see this, we write Eq. (1) with $P^{f}=\mathrm{E}(\tilde{P})$ as

$$
\tilde{\Pi}=\tilde{Z}\{W+\mathrm{E}(\tilde{P}) Q-C(Q)+[\tilde{P}-\mathrm{E}(\tilde{P})](Q-X)\}
$$

It is evident from Eq. (15) that an over-hedge decreases (increases) the firm's nominal income at date 1 as $P$ increases (decreases), which is more likely when $Z$ is lower (higher).

\footnotetext{
${ }^{6}$ If $R(\Pi)=1$ for all $\Pi>0$, i.e, the firm has a logarithmic utility function, the firm's optimal forward position is a full-hedge, i.e., $X^{*}=Q^{*}$.
} 
Given risk aversion, the over-hedge is more effective in reducing the variability of $U^{\prime}\left(\tilde{\Pi}^{*}\right) \tilde{Z}$. Since the elasticity of the firm's marginal utility is gauged by the Arrow-Pratt measure of relative risk aversion, $R(\Pi)=-\Pi U^{\prime \prime}(\Pi) / U^{\prime}(\Pi)$, the firm's marginal utility is insensitive (sensitive) to the price risk if $R(\Pi)$ is small (large). The cross-hedging incentive is therefore stronger (weaker) if $R(\Pi)$ is small (large). Taking expectations on both sides of Eq. (15) yields

$$
\mathrm{E}(\tilde{\Pi})=W+\mathrm{E}(\tilde{P}) Q-C(Q)+\operatorname{Cov}(\tilde{P}, \tilde{Z})(Q-X)
$$

As is evident from the last term on the right-hand side of Eq. (16), an over-hedge increases the firm's expected real income at date 1 since $\operatorname{Cov}(\tilde{P}, \tilde{Z})<0$. This gives rise to a speculative incentive that induces the firm to opt for an over-hedge. This speculative incentive is stronger (weaker) if the firm is less (more) risk averse, which dominates (is dominated by) the cross-hedging incentive, thereby rendering the optimality of an over-hedge (under-hedge), if $R(\Pi) \leq(\geq) 1$ for all $\Pi>0$.

\section{Optimal production decision}

In this section, we examine the firm's optimal production decision. Substituting Eq. (4) with $P^{f}=\mathrm{E}(\tilde{P})$ into Eq. (3) yields $C^{\prime}\left(Q^{*}\right)=\mathrm{E}(\tilde{P})$, which implies that the separation theorem holds under the joint price and inflation risk. If the firm cannot hedge against the price risk, i.e., $X \equiv 0$, the first-order condition for program (2) becomes

$$
\mathrm{E}\left\{U^{\prime}\left\{\tilde{Z}\left[W+\tilde{P} Q^{\circ}-C\left(Q^{\circ}\right)\right]\right\} \tilde{Z}\left[\tilde{P}-C^{\prime}\left(Q^{\circ}\right)\right]\right\}=0
$$

where $Q^{\circ}$ is the optimal output level when forward trading is not allowed. Differentiating $\mathrm{E}[U(\tilde{\Pi})]$ with respect to $Q$ and evaluating the resulting derivative at $Q=Q^{*}$ and $X=0$ yields

$$
\left.\frac{\partial \mathrm{E}[U(\tilde{\Pi})]}{\partial Q}\right|_{Q=Q^{*}, X=0}=\mathrm{E}\left\{U^{\prime}\left\{\tilde{Z}\left[W+\tilde{P} Q^{*}-C\left(Q^{*}\right)\right]\right\} \tilde{Z}[\tilde{P}-\mathrm{E}(\tilde{P})]\right\},
$$

since $C^{\prime}\left(Q^{*}\right)=\mathrm{E}(\tilde{P})$. If the right-hand side of Eq. (18) is negative (positive), it follows immediately from Eq. (17) and the second-order conditions for program (2) that $Q^{\circ}<(>$ 
) $Q^{*}$.

Differentiating $\mathrm{E}[U(\tilde{\Pi})]$ with respect to $X$ and evaluating the resulting derivative at $Q=Q^{*}$ and $X=0$ yields

$$
\left.\frac{\partial \mathrm{E}[U(\tilde{\Pi})]}{\partial X}\right|_{Q=Q^{*}, X=0}=\mathrm{E}\left\{U^{\prime}\left\{\tilde{Z}\left[W+\tilde{P} Q^{*}-C\left(Q^{*}\right)\right]\right\} \tilde{Z}[\mathrm{E}(\tilde{P})-\tilde{P}]\right\}
$$

If $X^{*}>(<) 0$, it follows from Eq. (4) and the second-order conditions for program (2) that the right-hand side of Eq. (19) is positive (negative). Eqs. (18) and (19) then imply that $Q^{\circ}<(>) Q^{*}$ if $X^{*}>(<)$, thereby invoking the following proposition.

Proposition 2 If the competitive firm optimally sells (purchases) its output forward, i.e., $X^{*}>(<) 0$, at the unbiased forward price, $P^{f}=\mathrm{E}(\tilde{P})$, under the joint price and inflation risk, banning the firm from forward trading induces the firm to lower (raise) its optimal output level, i.e., $Q^{\circ}<(>) Q^{*}$.

From Proposition $1, X^{*}>Q^{*}$ if $R(\Pi) \leq 1$ for all $\Pi>0$. In this case, $Q^{\circ}<Q^{*}$ since $X^{*}>0$. On the other hand, $X^{*}<Q^{*}$ if $R(\Pi) \geq 1$ for all $\Pi>0$. In this case, $X^{*}$ can be positive or negative, and thus $Q^{\circ}$ can be smaller or greater than $Q^{*}$, respectively. These results are consistent with those of Adam-Müller (2002).

To see the intuition for Proposition 2, we recast Eq. (17) as

$$
C^{\prime}\left(Q^{\circ}\right)=\mathrm{E}(\tilde{P})+\frac{\operatorname{Cov}\left\{U^{\prime}\left\{\tilde{Z}\left[W+\tilde{P} Q^{\circ}-C\left(Q^{\circ}\right)\right]\right\} \tilde{Z}, \tilde{P}\right\}}{\mathrm{E}\left\{U^{\prime}\left\{\tilde{Z}\left[W+\tilde{P} Q^{\circ}-C\left(Q^{\circ}\right)\right]\right\} \tilde{Z}\right\}} .
$$

Eq. (20) states that the firm's optimal output level, $Q^{\circ}$, is the one that equates the marginal cost of production, $C^{\prime}\left(Q^{\circ}\right)$, to the certainty equivalent output price that takes the inflation risk and the firm's preferences into account. Indeed, the second term on the right-hand side of Eq. (20) captures the price risk premium, which must be positive (negative) if the firm optimally sells (purchases) its output forward, i.e., $X^{*}>(<) 0$, at the unbiased forward price, $P^{f}=\mathrm{E}(\tilde{P})$, thereby implying that $Q^{\circ}<(>) Q^{*}$. 
In the absence of the inflation risk, i.e., $\tilde{Z} \equiv 1$, the price risk premium is unambiguously negative since $U^{\prime \prime}(\Pi)<0$. In this case, $X^{*}>0$ and thus $Q^{\circ}<Q^{*}$, which is the well-known result of Holthausen (1979). When the inflation risk prevails, the price risk premium can be positive or negative. Since the elasticity of the firm's marginal utility is gauged by the Arrow-Pratt measure of relative risk aversion, $R(\Pi)=-\Pi U^{\prime \prime}(\Pi) / U^{\prime}(\Pi)$, the firm's marginal utility is insensitive to the price risk if $R(\Pi)$ is small. In this case, the price risk premium is mainly driven by the covariance between $\tilde{P}$ and $\tilde{Z}$, which is negative. Hence, the firm optimally produces less if $R(\Pi) \leq 1$ for all $\Pi>0$. To see that the price risk premium can be

positive if $R(\Pi)$ is large, consider the case that $\tilde{Z}=1 / \tilde{P}$. The firm's real income at date 1 is then given by $Q^{\circ}+\left[W-C\left(Q^{\circ}\right)\right] / P$, which decreases (increases) as $P$ increases (decreases). Given risk aversion, the firm's marginal utility is positively correlated with the price risk. The multiple of the firm's marginal utility and the inflation risk is also positively correlated with the price risk if $R(\Pi) \geq 1+A(\Pi) Q^{\circ}$ for all $\Pi>0$, where $A(\Pi)=-U^{\prime \prime}(\Pi) / U^{\prime}(\Pi)$ is the Arrow-Pratt measure of absolute risk aversion. This gives rise to a positive price risk premium so that the firm optimally produces more when forward trading is banned.

\section{Conclusion}

In this paper, we examine the behavior of a competitive firm facing joint inflation and price risk. When the price risk is negatively correlated with the inflation risk in the sense of expectation dependence, we show that the firm optimally opts for an over-hedge (underhedge) if the firm's coefficient of relative risk aversion is everywhere no greater (no smaller) than unity. We show further that banning the firm from forward trading may induce the firm to produce more or less, depending on whether the price risk premium is positive or negative, respectively. While the price risk premium is unambiguously negative in the absence of the inflation risk, it is not the case when the inflation risk prevails. In contrast to the conventional wisdom, forward hedging needs not always promote production should firms take inflation seriously. 


\section{References}

Adam-Müller AFA (2002a) What to do if a dollar is not a dollar? The impact of inflation risk on production and risk management. Journal of Futures Markets 22:371-386

Adam-Müller AFA (2002b) Hedging price risk when real wealth matters. Journal of International Money and Finance 19:549-560

Battermann HL, Broll U (2001) Inflation risk, hedging, and exports. Review of Development Economics 5:355-362

Broll U, Welzel P, Wong KP (2015) Futures hedging with basis risk and expectation dependence. International Review of Economics 62:213-221

Cuadras CM (2002) On the covariance between functions. Journal of Multivariate Analysis $81: 19-27$

Holthausen DM (1979) Hedging and the competitive firm under price uncertainty. American Economic Review 69:989-995.

Li J (2011) The demand for a risky asset in the presence of a background risk. Journal of Economic Theory 146:372-391

Sandmo A (1971) On the theory of the competitive firm under price uncertainty. American Economic Review 61:65-73

Wong KP (2012) Production and hedging under state-dependent preferences. Journal of Futures Markets 32:945-963.

Wong KP (2013) A note on exports and hedging exchange rate risks: the multi-country case. Journal of Futures Markets 33:1191-1196

Wright R (1987) Expectation dependence of random variables, with an application in portfolio theory. Theory and Decision 22:111-124 